\title{
VARIABILIDADE E CARACTERIZAÇÃO DE FRUTOS DE PITANGUEIRAS EM MUNICÍPIOS BAIANOS ${ }^{1}$
}

\author{
ALEX BATISTA DIAS², MARIA ANGÉLICA PEREIRA DE CARVALHO ${ }^{3}$, \\ ANA CRISTINA VELLO LOYOLA DANTAS ${ }^{4}$, VALDIR JOSÉ DE ALMEIDA FONSECA ${ }^{5}$
}

RESUMO- O presente trabalho teve como objetivo obter informações sobre as características morfológicas dos frutos das pitangueiras localizadas em cinco municípios do Estado da Bahia. Os genótipos foram identificados, georreferenciados com o auxílio de GPS e de cada genótipo foram coletados 30 frutos no estádio de maturação fisiológica, avaliando-se: massa, comprimento e diâmetro do fruto, massas da polpa e das sementes, $\mathrm{pH}$, acidez titulável, teor de sólidos solúveis e relação SS/AT. Os dados foram submetidos à análise descritiva, obtendo-se medidas de centralidade e de dispersão, correlação linear entre os caracteres e análise multivariada de agrupamento. Os resultados revelaram a existência de variabilidade para a maioria das características avaliadas, em especial para a massa do fruto, da semente e da polpa. Houve a formação de cinco grupos principais de dissimilaridade genética para a população geral, sendo a menor distância genética $(0,22)$ verificada entre os genótipos IN3 e IN8 provenientes do município de Inhambupe. Os genótipos apresentam frutos com características de interesse para exploração comercial com porcentagem de polpa acima de $68 \%$, sendo possível a identificação de materiais que reúnem altos valores para massa do fruto, massa da polpa, sólidos solúveis (SS), acidez titulável (AT) e SS/AT.

Termos para indexação: Eugenia uniflora, dissimilaridade genética, variabilidade.

\section{VARIABILITY AND CHARACTERIZATION OF FRUITS OF SURINAM CHERRY IN MUNICIPALITIES IN THE STATE OF BAHIA}

\begin{abstract}
This study aimed to obtain information on the morphological characteristics of fruits of Surinam cherry located in five districts of the State of Bahia. The genotypes were identified, demarcated with the help of GPS and from each genotype, 30 fruits at maturity stage were collected, being evaluated: weight, length and diameter of fruit, pulp and seed masses, $\mathrm{pH}$, acidity, soluble solids and SS / TA.). The data were statistically analyzed by obtaining measures of centrality and dispersion, linear correlation between the characters and multivariate clustering. The results revealed the existence of variability for most traits, particularly for the fruit, seed and pulp masses. There was formation of five major groups of genetic similarity for the general population, with the smallest genetic distance $(0.22)$ observed between the genotypes IN3 and IN8 from the municipality of Inhambupe. The genotypes have fruit with interesting characteristics for commercial exploitation with pulp percentage above $68 \%$, being possible to identify materials that gather high values for fruit mass, pulp mass, soluble solids (SS), titratable acidity (TA) and SS / TA.
\end{abstract}

Index terms: Eugenia uniflora, genetic dissimilarity, variability.

\footnotetext{
'(Trabalho 057-11). Recebido em: 12-01-2011. Aceito para publicação em: 22-06-2011.

${ }^{2}$ Eng. Agrônomo, Msc. Em Ciências Agrárias. Centro de Ciências Agrárias, Ambientais e Biológicas (CCAAB) - Universidade Federal do Recôncavo da Bahia (UFRB). Campus Universitário, Cruz das Almas, BA - 44380-000. E-mail: a.batista.dias@bol.com.br. ${ }^{3}$ Eng. Agrônoma, Prof. Dr ${ }^{\mathrm{a}}$ em Fitotecnia - CCAAB/UFRB. E-mail: mapcosta@ufba.br

${ }^{4}$ Eng. Agrônoma, Prof. Dr ${ }^{\mathrm{a}}$ em Melhoramento Genético de Plantas - CCAAB/UFRB. E-mail: acloyola@ufrb.edu.br

${ }^{5}$ Eng. Agrônomo, Dr em Ciências Agrárias - CCAAB/UFRB. E-mail: valdir-fonseca@bol.com.br
} 


\section{INTRODUÇÃO}

Existe uma tendência mundial de consumo de frutas tropicais que pode ser atribuída ao valor nutricional, às características organolépticas e à necessidade de melhoria na qualidade de vida das pessoas associada a aspectos de saúde e bem-estar (OLIVEIRA, 2009). Vários municípios da Bahia apresentam grande diversidade de frutas tropicais nativas e exóticas, em virtude das condições edafoclimáticas favoráveis aos cultivos.

A pitangueira (Eugenia uniflora L.) é uma fruteira nativa do Brasil e encontra-se distribuída, praticamente, por todo o território nacional. Quando cultivada, mesmo em pequenos pomares, pode possibilitar uma atividade econômica promissora, devido ao sabor agradável e refrescante de seus frutos, com um característico aroma atrativo.

O fruto é uma baga, sendo a polpa a principal parte comestível na forma in natura ou através da exploração agroindustrial para a obtenção da polpa integral congelada, suco engarrafado, fabricação de sorvete, picolé, licor, geleia, vinho, dentre outros, bastante apreciado pela população. Além de produto de consumo humano, a pitangueira pode fornecer madeira e servir como planta ornamental e cerca viva, por apresentar copa densa e compacta. Suas folhas são usadas na medicina popular na forma de chá para controlar a diarreia e combater a tosse (LIRA JUNIOR et al., 2007).

O peso médio de frutos é uma característica importante para o mercado de frutas frescas, uma vez que aqueles mais pesados são também os de maior tamanho, tornando-se mais atrativos para os consumidores. Os parâmetros químico e físicoquímico relacionados à acidez titulável e ao teor de sólidos solúveis da polpa são mais relevantes no que se refere à elaboração de subprodutos, mesmo porque a pitanga é uma fruta essencialmente voltada à industrialização (ARAÚJO, 1995).

A caracterização constitui uma das principais etapas dos trabalhos com germoplasma, pois permite indicar plantas com potencial de uso imediato, bem como identificar genótipos que apresentem características interessantes para o melhoramento, além de ser fundamental para o estabelecimento de formas de exploração econômica e racional (LACERDA et al., 2001). Sem o conhecimento das características agronômicas, morfológicas e citogenéticas, não é possível manipular adequadamente a variabilidade genética observada (MELETTI et al., 1992)

A caracterização morfológica consiste na aferição de caracteres botânicos herdáveis, facilmente visíveis e mensuráveis que, a princípio, podem ser expressos em todos os ambientes. Embora apresente limitações relacionadas aos caracteres com herança aditiva, os quais são altamente influenciados pelo ambiente, essa técnica é utilizada internacionalmente para caracterizar e avaliar distintos genótipos por meio da observação fenotípica dos caracteres (WEILER, 2006).

Todo caráter deve contribuir de alguma maneira para descrever a variabilidade; porém, à medida que cresce o número de descritores, aumenta também a possibilidade de eles serem redundantes ou altamente correlacionados (DAHER, 1993). A seleção combinada de vários caracteres por meio da análise multivariada permite discriminar, com mais eficiência, os materiais promissores. Dentre as técnicas disponíveis, têm-se as análises de agrupamento, e da distância Euclidiana média como medida de dissimilaridade (CRUZ, 1990). $\mathrm{O}$ conhecimento do modo como a variabilidade de uma espécie está distribuída nas suas populações é essencial para a sua conservação e manejo.

O presente trabalho teve como objetivo obter informações sobre a variabilidade de genótipos de pitangueira em municípios do Estado da Bahia, a partir da caracterização dos frutos, visando à identificação de materiais promissores para cultivo pelos produtores ou para futuros trabalhos de melhoramento da espécie.

\section{MATERIAL E MÉTODOS}

Foram avaliados 50 genótipos de pitangueira (Eugenia uniflora L.) em cinco populações localizadas em municípios na região do Recôncavo da Bahia (Cruz das Almas, Conceição do Almeida e Muritiba) e também no nordeste baiano (Alagoinhas e Inhambupe). Os municípios do Recôncavo baiano situam-se entre as coordenadas $12^{\circ} 37^{\prime}$ a $12^{\circ} 48^{\prime}$ de latitude sul e $38^{\circ} 59^{\prime}$ a $39^{\circ} 15^{\prime}$ de longitude oeste, com umidade relativa do ar de $81 \%$, precipitação mensal média de $95,2 \mathrm{~mm}$ e temperaturas que variam de $20,1^{\circ} \mathrm{C}$ a $28,7^{\circ} \mathrm{C}$. O município de Inhambupe está localizado a $11^{\circ} 47^{\prime}$ latitude sul e $38^{\circ} 21^{\prime}$ ' longitude oeste, e Alagoinhas a $12^{\circ} 10^{\prime}$ latitude sul e $38^{\circ} 22^{\prime}$ longitude oeste, região com umidade relativa do ar média de $74 \%$, precipitação mensal média de 76,4 $\mathrm{mm}$ e temperaturas que variam de 18 a $27^{\circ} \mathrm{C}$. Foram avaliadas plantas em idade adulta, provenientes de sementes, identificadas em áreas rurais, quintais urbanos e em pequena coleção da Estação Experimental da Empresa Baiana de Desenvolvimento Agrícola (EBDA), em Conceição de Almeida-BA.

Os genótipos foram identificados e georreferenciados com o auxílio de GPS. Para cada 
genótipo, foram coletados 30 frutos no estádio de maturação fisiológica, colocados em sacos plásticos, acondicionados em caixas de isopor e encaminhados ao Laboratório de Tecnologia Vegetal da URFB/Cruz das Almas - BA, para caracterização física quanto a: massa $(\mathrm{g})$, comprimento $(\mathrm{mm})$, diâmetro $(\mathrm{mm})$, massa da polpa (g), massa da semente $(\mathrm{g})$, percentagens de polpa e da semente. Medidas de comprimento foram tomadas entre o ápice e a inserção do pedúnculo (diâmetro longitudinal) e na porção mediana do fruto (diâmetro transversal), determinadas com o uso de um paquímetro digital, com precisão de duas casas decimais.

Para as determinações química (acidez titulável) e físico-químicas ( $\mathrm{pH}$ e sólido solúveis), as polpas foram retiradas e homogeneizadas manualmente, peneiradas, retirando-se três amostras para análise, conforme recomendação do Instituto Adolfo Lutz (1985).

Os dados foram analisados por estatística descritiva, com o uso do programa SAS - Statistical Analysis System (SAS Institute, 1989), obtendo-se medidas de centralidade e de dispersão (média desvio-padrão e coeficiente de variação). Como medida de dissimilaridade, calculou-se a distância euclidiana média e, para a formação dos agrupamentos, utilizouse o método UPGMA - Unweighted Pair Group Method with Arithmetic Mean (SNEATH; SOKAL, 1973). Foram calculadas as taxas de contribuição relativas para a dissimilaridade pelo método de Singh (1981). As análises foram realizadas pelo programa estatístico GENES (CRUZ, 2008), e o dendrograma foi obtido pelo programa STATISTICA(STATSOFT, 2005). Para a identificação de genótipos potenciais, foi utilizada a técnica de índice de soma de classificação de Mulamba e Mock (1978), considerando-se as variáveis de maior interesse para utilização industrial, a partir da caracterização dos frutos.

\section{RESULTADOS E DISCUSSÃO}

A caracterização morfológica dos frutos revelou a existência de variabilidade entre os genótipos, para a maioria das características avaliadas (Tabela 1).

A amplitude de variação para diâmetros transversal e longitudinal foi de 15,19 a $25,39 \mathrm{~mm}$ e $10,78 \mathrm{~mm}$ a $19,46 \mathrm{~mm}$, respectivamente, com maior coeficiente de variação para diâmetro transversal $(13,25 \%)$.

A massa do fruto apresentou variação ampla, de 1,28 a 6,52 g, com coeficiente de variação de 35,02 $\%$. A média encontrada de $2,79 \mathrm{~g}$ é inferior ao valor citado por Fonseca et al. (2009), que foi de 4,24 g em frutos provenientes de Cruz das Almas - BA. O genótipo que apresentou maior massa do fruto foi o IN9, com 6,52 g. Para comercialização in natura dos frutos, o peso médio é uma característica importante, pois os maiores frutos são os mais atrativos para os consumidores.

A massa da semente apresentou o intervalo de variação de 0,15 a 1,12 g, com média de $0,58 \mathrm{~g}$, e o maior coeficiente de variação $40,79 \%$, indicando uma ampla variabilidade. O percentual de semente variou de 11,82 a $31,77 \%$, com um coeficiente de variação de $23,88 \%$. O valor médio de $20,54 \%$ é semelhante ao obtido por Fonseca et al. (2009), em frutos avaliados em Cruz das Almas-BA.

O percentual de polpa apresentou uma amplitude de 68,23 a $88,13 \%$, com média de $79,46 \%$. Isto mostra que este caráter apresenta baixa variabilidade, confirmado pelo coeficiente de variação, que foi de $6,17 \%$. A média encontrada é semelhante ao valor relatado por Bezerra et al. (2004), de $80 \%$, e superior ao encontrada por Lopes (2005) e Fonseca et al. (2009), de $66,64 \%$ e $58,26 \%$, respectivamente. Quanto maior o percentual de polpa dos frutos, melhor, tanto para o consumo in natura quanto para a indústria.

Entre as variáveis química e físico-químicas avaliadas (Tabela 2), o $\mathrm{pH}$ apresentou menor variação, entre 2,61 e 3,07 (coeficiente de variação de $3,31 \%$ ). No processamento de frutos, valores de pH baixos favorecem a conservação dos alimentos, não havendo necessidade de adição de ácido na formulação para evitar o crescimento de leveduras, dificultando o desenvolvimento de microrganismos (LIMA et al., 2002). Por outro lado, valores mais altos de $\mathrm{pH}$ são preferidos para o consumo in natura. Neste trabalho, todos os genótipos apresentaram $\mathrm{pH}$ com valor recomendado por Brasil (1999) para polpa de pitanga, entre 2,5 e 3,4 .

O teor de sólidos solúveis (SS) variou de 9,0 a $15,30^{\circ}$ Brix, com média de $10,88^{\circ}$ Brix. A média geral está acima da encontrada por Oliveira et al. (2006) para pitanga, em Selvíria-MS, 7,0 ${ }^{\circ}$ Brix. Para o resultado encontrado, todos os genótipos estão dentro dos valores estabelecidos por Brasil (1999) para polpa de pitanga, cujo valor mínimo exigido é de $6,0^{\circ}$ Brix, o que coloca essa espécie em excelente posição para a industrialização de seus frutos. Frutos com altos teores de sólidos solúveis são geralmente preferidos, tanto para o consumo in natura quanto para industrialização, por oferecerem a vantagem de propiciar maior rendimento no processamento, em razão da maior quantidade de néctar produzido por quantidade de polpa.

Os genótipos avaliados apresentaram frutos com acidez titulável (AT) variando de 1,40 a 2,34\%, 
com média de $1,87 \%$, ligeiramente inferior aos observados por Oliveira et al. (2006), em Campina Grande-PB, de 2,23\%, mas que atendem às exigências de Brasil (1999) para polpa de pitanga (mínimo de $0,92 \%$ ). A acidez constitui uma variável de grande interesse para o estado de conservação de produtos alimentícios. Para Lima et al. (2002) e Pinto et al. (2003), pode-se considerar que os genótipos com acidez titulável acima de 1,00\% são os de maior interesse para a agroindústria, tendo em vista não haver necessidade da adição de ácido para a conservação da polpa, que é um artifício utilizado para dificultar o desenvolvimento de microrganismos.

Para a relação SS/AT, os valores variaram de 4,54 a 7,31, com média de 5,85, estando próximo ao resultado obtido por Bárbara et al. (2010) em frutos de pitangueira, no município de Santo Antônio de Jesus-BA $(4,48)$. A relação SS/AT é mais adequada para avaliar o sabor e o ponto de maturação do que a medição isolada de açúcares e de acidez, além de ser um importante parâmetro para avaliar a qualidade dos frutos, constituindo uma das formas mais usuais de se avaliar o sabor e os produtos obtidos (LIMA et al., 2002).

$\mathrm{O}$ estudo de correlações entre caracteres é uma importante ferramenta para programas de melhoramento genético, quando se objetiva aprimorar o material para um conjunto de caracteres simultaneamente. Assim, altos valores de correlação entre características indicam a possibilidade de realização de trabalhos de seleção para fatores de difícil observação, baseando-se na análise de fatores facilmente observáveis (SANTOS, 2010). Coeficientes de correlação linear positivos e significativos foram obtidos para as associações entre os caracteres físicos, com valor de " $\mathrm{r}$ " acima de $70 \%$. Não foram observadas correlações significativas entre os caracteres físicos e químicos, e físico-químicos e entre o químico e os físico-químicos, apenas a correlação entre SS/AT e pH, merece destaque por apresentar coeficiente de correlação significativo de 0,68 (Tabela 3).

Baseado no conjunto de características físicas, química e físico-químicas analisadas, elaborouse o dendrograma da dissimilaridade genética, no qual se observa a formação dos grupos distintos que apresentaram algum grau de dissimilaridade (Figura 1). Neste trabalho, assumiu-se como ponto de corte no dendrograma a similaridade genética média de 1,26 entre todos os genótipos em estudo, verificando-se a formação de cinco grupos, sendo o grupo 5 subdividido em vários subgrupos. A menor distância genética verificada foi de 0,22 , entre os genótipos IN3 e IN8 provenientes do município de Inhambupe, provavelmente por estes genótipos serem originados da mesma matriz. A maior distância genética verificada foi de 4,08, entre os genótipos IN9 e MU8, sendo que o primeiro foi coletado no município de Inhambupe, e o segundo, em Muritiba. Estes municípios são distantes entre si, o que diminui a chance de estas plantas serem aparentadas.

O coeficiente de correlação cofenético (CCC) entre a matriz de distância genética e a matriz de agrupamento foi positiva, com valor de $82,70 \%$, considerado de boa magnitude (VAZ PATTO et al., 2004), permitindo fazer inferências sobre o dendrograma.

A variável que mais contribuiu para a dissimilaridade genética e, consequentemente, para a formação dos grupos foi o diâmetro transversal do fruto $(38,17 \%)$, seguida pelo diâmetro longitudinal do fruto $(30,57 \%)$ e de sólidos solúveis $(12,10 \%)$. Por outro lado, as variáveis que menos contribuíram para a divergência genética foram o $\mathrm{pH}(0,07 \%)$ e a acidez titulável (0,39\%) (Tabela 4).

A comprovação de variabilidade entre os genótipos é um indício de origens genéticas distintas, o que poderá ser mais bem investigado com a caracterização com o uso de marcadores moleculares. Esta variabilidade, uma vez confirmada, poderá ser fixada por método de propagação vegetativa e utilizada para a conservação em bancos ativos de germoplasma (BAG) e em programas de melhoramento genético.

Através da técnica de índice de soma de classificação de Mulamba e Mock (1978), em que se consideraram altos valores para as variáveis massa do fruto, massa da polpa, percentagem de polpa, SS, acidez e SS/AT e baixo pH, os genótipos IN9, CZ9, AL3, CA8, CZ5, CA1, AL8, IN1, AL4 e CA2 podem ser indicados para aproveitamento industrial. 
TABELA 1 - Valores médios referentes a caracteres físicos dos frutos de 50 pitangueiras (Eugenia uniflora L.) provenientes de municípios da Bahia - 2010.

\begin{tabular}{|c|c|c|c|c|c|c|c|}
\hline GEN & DTF & DLF & MF & MS & MP & $\% \mathrm{P}$ & $\% \mathrm{~S}$ \\
\hline CZ1 & 19,02 & 14,51 & 2,76 & 0,62 & 2,14 & 77,66 & 22,34 \\
\hline CZ2 & 17,53 & 13,56 & 2,67 & 0,72 & 1,95 & 73,18 & 26,82 \\
\hline CZ3 & 18,39 & 13,18 & 2,65 & 0,77 & 1,89 & 71,14 & 28,86 \\
\hline CZ4 & 19,05 & 14,57 & 2,95 & 0,56 & 2,38 & 80,84 & 19,16 \\
\hline CZ5 & 21,09 & 14,52 & 3,78 & 0,83 & 2,94 & 77,94 & 22,06 \\
\hline CZ6 & 19,88 & 14,08 & 2,99 & 0,78 & 2,21 & 73,98 & 26,02 \\
\hline CZ7 & 16,82 & 12,17 & 1,77 & 0,33 & 1,44 & 81,20 & 18,80 \\
\hline CZ8 & 20,03 & 14,42 & 2,69 & 0,50 & 2,19 & 81,33 & 18,67 \\
\hline CZ9 & 23,88 & 18,11 & 4,72 & 0,75 & 3,97 & 84,09 & 15,91 \\
\hline CZ10 & 17,75 & 13,06 & 2,06 & 0,65 & 1,41 & 68,23 & 31,77 \\
\hline CA1 & 19,72 & 14,20 & 2,92 & 0,53 & 2,39 & 81,84 & 18,16 \\
\hline CA2 & 20,47 & 14,48 & 2,99 & 0,55 & 2,44 & 81,77 & 18,23 \\
\hline CA3 & 21,13 & 15,61 & 2,93 & 0,63 & 2,30 & 78,39 & 21,61 \\
\hline CA4 & 21,14 & 15,33 & 3,42 & 0,57 & 2,85 & 83,45 & 16,55 \\
\hline CA5 & 17,16 & 11,96 & 1,64 & 0,21 & 1,43 & 87,29 & 12,71 \\
\hline CA6 & 18,88 & 14,65 & 2,77 & 0,54 & 2,23 & 80,61 & 19,39 \\
\hline CA7 & 17,85 & 13,28 & 2,16 & 0,48 & 1,68 & 77,78 & 22,22 \\
\hline CA8 & 19,14 & 14,06 & 2,88 & 0,49 & 2,39 & 83,13 & 16,87 \\
\hline CA9 & 16,50 & 12,36 & 2,21 & 0,39 & 1,82 & 82,29 & 17,71 \\
\hline CA10 & 17,61 & 12,83 & 1,78 & 0,41 & 1,37 & 77,21 & 22,79 \\
\hline AL1 & 17,48 & 11,83 & 1,84 & 0,22 & 1,64 & 88,18 & 11,82 \\
\hline AL2 & 18,99 & 13,71 & 2,60 & 0,66 & 1,94 & 74,65 & 25,35 \\
\hline AL3 & 19,30 & 14,69 & 2,86 & 0,42 & 2,44 & 85,16 & 14,84 \\
\hline AL4 & 18,33 & 13,87 & 2,37 & 0,40 & 1,97 & 83,15 & 16,85 \\
\hline AL5 & 18,68 & 13,86 & 2,19 & 0,46 & 1,73 & 78,84 & 21,16 \\
\hline AL6 & 18,07 & 13,18 & 2,10 & 0,44 & 1,66 & 79,11 & 20,89 \\
\hline AL7 & 19,05 & 14,02 & 2,39 & 0,53 & 1,86 & 77,90 & 22,10 \\
\hline AL8 & 22,44 & 14,95 & 3,84 & 0,75 & 3,09 & 80,55 & 19,45 \\
\hline AL9 & 17,47 & 11,83 & 1,76 & 0,25 & 1,51 & 85,58 & 14,42 \\
\hline AL10 & 17,31 & 12,46 & 1,86 & 0,41 & 1,45 & 77,86 & 22,14 \\
\hline IN1 & 20,28 & 14,40 & 3,18 & 0,84 & 2,34 & 73,47 & 26,53 \\
\hline IN2 & 22,01 & 16,88 & 4,10 & 0,90 & 3,20 & 78,08 & 21,92 \\
\hline IN3 & 20,63 & 16,05 & 3,26 & 0,77 & 2,49 & 76,38 & 23,62 \\
\hline IN4 & 21,14 & 15,94 & 3,37 & 0,69 & 2,68 & 79,46 & 20,54 \\
\hline IN5 & 21,55 & 16,96 & 3,60 & 1,09 & 2,51 & 69,60 & 30,40 \\
\hline IN6 & 20,04 & 15,70 & 2,70 & 0,82 & 1,88 & 69,77 & 30,23 \\
\hline IN7 & 23,73 & 19,46 & 5,42 & 1,12 & 4,30 & 79,37 & 20,63 \\
\hline IN8 & 20,90 & 16,26 & 3,53 & 0,86 & 2,67 & 75,51 & 24,49 \\
\hline IN9 & 25,39 & 19,24 & 6,52 & 1,10 & 5,42 & 83,12 & 16,88 \\
\hline IN10 & 18,67 & 13,95 & 2,43 & 0,65 & 1,78 & 73,19 & 26,81 \\
\hline MU1 & 16,98 & 12,37 & 2,00 & 0,28 & 1,72 & 85,99 & 14,01 \\
\hline MU2 & 18,87 & 13,48 & 2,53 & 0,43 & 2,10 & 83,21 & 16,79 \\
\hline MU3 & 17,47 & 12,98 & 2,23 & 0,42 & 1,81 & 80,21 & 19,79 \\
\hline MU4 & 21,47 & 15,21 & 3,77 & 0,80 & 2,97 & 78,75 & 21,25 \\
\hline MU5 & 19,89 & 14,63 & 3,04 & 0,81 & 2,23 & 73,32 & 26,68 \\
\hline MU6 & 16,16 & 11,24 & 1,63 & 0,21 & 1,42 & 86,93 & 13,07 \\
\hline MU7 & 19,56 & 14,74 & 2,76 & 0,53 & 2,23 & 80,94 & 19,06 \\
\hline MU8 & 15,19 & 10,78 & 1,28 & 0,15 & 1,13 & 87,98 & 12,02 \\
\hline MU9 & 16,32 & 11,55 & 1,69 & 0,25 & 1,44 & 85,31 & 14,69 \\
\hline MU10 & 18,44 & 12,19 & 2,16 & 0,48 & 1,68 & 78,00 & 22,00 \\
\hline Média & 19,27 & 14,18 & 2,79 & 0,58 & 2,21 & 79,46 & 20,54 \\
\hline CV (\%) & 10,89 & 13,25 & 35,02 & 40,79 & 35,67 & 6,17 & 23,88 \\
\hline D. P. & 2,10 & 1,88 & 0,98 & 0,24 & 0,79 & 4,90 & 4,90 \\
\hline
\end{tabular}

$\mathrm{DLF}=$ Diâmetro longitudinal do fruto $(\mathrm{mm}) ; \mathrm{DTF}=$ diâmetro transversal do fruto $(\mathrm{mm}) ; \mathrm{MF}=$ massa do fruto $(\mathrm{g}) ; \mathrm{MS}=\mathrm{massa}$ da semente $(\mathrm{g}), \mathrm{MP}=$ massa da polpa $(\mathrm{g}), \% \mathrm{P}=$ porcentagem de polpa, $\% \mathrm{~S}=$ porcentagem de semente; $\mathrm{IN}=\mathrm{Inhambupe} ; \mathrm{CA}=\mathrm{Conceição}$ do Almeida; $\mathrm{CZ}=$ Cruz das Almas; $\mathrm{UM}=$ Muritiba; $\mathrm{AL}=$ Alagoinhas; $\mathrm{CV}=$ coeficiente de variação; $\mathrm{D}$. P .= desvio- padrão. 
TABELA 2 - Valores médios referentes a caracteres químicos e físico-químicos dos frutos de 50 pitangueiras (Eugenia uniflora L.) provenientes de municípios da Bahia - 2010. Cruz das Almas - BA.

\begin{tabular}{|c|c|c|c|c|}
\hline GEN & $\mathrm{pH}$ & SS & AT & SS/AT \\
\hline CZ1 & 2,74 & 11,00 & 2,07 & 5,31 \\
\hline CZ2 & 2,74 & 12,00 & 1,93 & 6,21 \\
\hline CZ3 & 2,84 & 11,50 & 1,82 & 6,26 \\
\hline CZ4 & 2,79 & 11,20 & 1,80 & 6,22 \\
\hline CZ5 & 2,63 & 10,90 & 2,34 & 4,65 \\
\hline CZ6 & 2,61 & 10,20 & 2,16 & 4,68 \\
\hline CZ7 & 2,75 & 11,50 & 1,86 & 4,54 \\
\hline CZ8 & 2,69 & 10,63 & 2,33 & 4,54 \\
\hline CZ9 & 2,74 & 11,30 & 2,01 & 5,62 \\
\hline CZ10 & 2,75 & 11,00 & 1,73 & 6,35 \\
\hline CA1 & 2,71 & 11,00 & 1,97 & 5,58 \\
\hline CA2 & 2,65 & 10,00 & 2,01 & 4,97 \\
\hline CA3 & 2,71 & 10,00 & 1,62 & 6,17 \\
\hline CA4 & 2,76 & 9,40 & 1,59 & 5,91 \\
\hline CA5 & 2,90 & 13,00 & 1,88 & 6,65 \\
\hline CA6 & 2,74 & 10,00 & 1,89 & 6,25 \\
\hline CA7 & 2,83 & 10,00 & 1,79 & 6,57 \\
\hline CA8 & 2,78 & 11,90 & 1,82 & 6,53 \\
\hline CA9 & 2,86 & 12,00 & 1,72 & 6,97 \\
\hline CA10 & 2,75 & 11,00 & 1,97 & 5,58 \\
\hline AL1 & 2,76 & 9,20 & 1,82 & 5,05 \\
\hline AL2 & 2,82 & 11,00 & 1,73 & 6,35 \\
\hline AL3 & 2,71 & 11,00 & 2,14 & 5,13 \\
\hline AL4 & 2,79 & 11,80 & 1,88 & 6,37 \\
\hline AL5 & 2,83 & 11,00 & 1,78 & 6,18 \\
\hline AL6 & 2,84 & 10,00 & 1,60 & 6,25 \\
\hline AL7 & 2,85 & 10,00 & 1,63 & 6,13 \\
\hline AL8 & 2,76 & 10,90 & 2,15 & 5,07 \\
\hline AL9 & 2,82 & 10,70 & 1,86 & 5,75 \\
\hline AL10 & 2,90 & 11,50 & 1,64 & 7,01 \\
\hline IN1 & 2,77 & 12,40 & 1,86 & 6,66 \\
\hline IN2 & 3,07 & 10,20 & 1,46 & 6,98 \\
\hline IN3 & 2,91 & 10,10 & 1,84 & 5,48 \\
\hline IN4 & 2,85 & 9,90 & 1,77 & 5,59 \\
\hline IN5 & 2,78 & 11,00 & 1,98 & 5,55 \\
\hline IN6 & 2,77 & 11,00 & 1,98 & 5,55 \\
\hline IN7 & 3,05 & 10,10 & 1,40 & 7,21 \\
\hline IN8 & 2,89 & 9,80 & 1,82 & 5,38 \\
\hline IN9 & 2,96 & 15,30 & 2,09 & 7,31 \\
\hline IN10 & 2,87 & 12,10 & 1,96 & 6,17 \\
\hline MU1 & 2,80 & 11,90 & 2,01 & 5,92 \\
\hline MU2 & 2,79 & 10,10 & 1,58 & 6,39 \\
\hline MU3 & 2,73 & 9,80 & 1,86 & 5,29 \\
\hline MU4 & 2,67 & 9,00 & 1,63 & 5,52 \\
\hline MU5 & 2,71 & 10,10 & 1,78 & 5,67 \\
\hline MU6 & 2,70 & 10,00 & 2,12 & 4,72 \\
\hline MU7 & 2,81 & 10,10 & 1,64 & 6,15 \\
\hline MU8 & 2,82 & 10,10 & 2,03 & 4,97 \\
\hline MU9 & 2,77 & 10,00 & 1,99 & 5,02 \\
\hline MU10 & 2,88 & 14,20 & 2,31 & 6,14 \\
\hline Média & 2,79 & 10,88 & 1,87 & 5,85 \\
\hline CV (\%) & 3,31 & 10,88 & 11,28 & 12,36 \\
\hline D. P. & 0,09 & 1,18 & 0,21 & 0,72 \\
\hline
\end{tabular}

$\mathrm{pH}=$ Potencial hidrogeniônico; $\mathrm{SS}=$ sólidos solúveis $\left({ }^{\circ} \mathrm{Brix}\right) ; \mathrm{AT}=$ acidez titulável (\% de ácido cítrico); $\mathrm{SS} / \mathrm{AT}=$ relação sólidos solúveis $/$ acidez titulável; $\mathrm{IN}=$ Inhambupe; $\mathrm{CA}=$ Conceição do Almeida; $\mathrm{CZ}=$ Cruz das Almas; $\mathrm{MU}=$ Muritiba; $\mathrm{AL}=\mathrm{Alagoinh}$ as; $\mathrm{C} . \mathrm{V} .=$ coeficiente de variação; D. P.= desvio-padrão. 
TABELA 3 - Coeficientes de correlação linear entre nove características avaliadas em frutos de pitangueira (Eugenia uniflora L.) de 50 genótipos provenientes de municípios situados no Estado da Bahia. Cruz das Almas - 2010.

\begin{tabular}{ccccccccc}
\hline & MF & MS & MP & DTF & DLF & pH & SS & AT \\
\hline MS & $0,84^{* *}$ & & & & & & & \\
MP & $0,99^{* *}$ & $0,74^{* *}$ & & & & & & \\
DTF & $0,95^{* *}$ & $0,83^{* *}$ & $0,93^{* *}$ & & & & & \\
DLF & $0,93^{* *}$ & $0,85^{* *}$ & $0,90^{* *}$ & $0,94^{* *}$ & & & & \\
pH & $0,24^{\text {ns }}$ & $0,20^{\text {ns }}$ & $0,24^{\text {ns }}$ & $0,16^{\text {ns }}$ & $0,27^{\text {ns }}$ & & & \\
SS & $0,16^{\text {ns }}$ & $0,10^{\text {ns }}$ & $0,16^{\text {ns }}$ & $0,06^{\text {ns }}$ & $0,03^{\text {ns }}$ & $0,28^{*}$ & & \\
AT & $-0,06^{\text {ns }}$ & $-0,10^{\text {ns }}$ & $-0,05^{\text {ns }}$ & $-0,06^{\text {ns }}$ & $-0,18^{\text {ns }}$ & $-0,47^{*}$ & $0,37^{* *}$ & \\
SS/AT & $0,24^{\text {ns }}$ & $0,23^{\text {ns }}$ & $0,23^{\text {ns }}$ & $0,15^{\text {ns }}$ & $0,25^{\text {ns }}$ & $0,68^{* *}$ & $0,41^{* *}$ & $-0,59^{* *}$ \\
\hline
\end{tabular}

**, *: Significativo a 1 e $5 \%$ de probabilidade pelo teste $\mathrm{t}$, respectivamente; ns - correlação não significativa. MF = massa do fruto, $\mathrm{MS}=$ massa da semente, $\mathrm{MP}=$ massa da polpa, $\mathrm{DLF}=$ Diâmetro longitudinal do fruto, $\mathrm{DTF}=$ diâmetro transversal do fruto, $\mathrm{pH}=$ Potencial hidrogeniônico, $\mathrm{SS}=$ sólidos solúveis e $\mathrm{AT}$ = acidez titulável.

TABELA 4- Contribuição relativa das características físicas, química e físico-químicas de 50 pitangueiras (Eugenia uniflora L.) provenientes de municípios situados no Estado da Bahia.

\begin{tabular}{lcc}
\hline Característica & S.j & VALOR (\%) \\
\hline Massa do Fruto (MF) & 2347,40 & 8,29 \\
Massa da Semente (MS) & 137,57 & 0,49 \\
Massa da Polpa (MP) & 1528,70 & 5,40 \\
Diâmetro transversal do fruto (DLF) & 10809,67 & 38,17 \\
Diâmetro longitudinal do fruto (DTF) & 8656,50 & 30,57 \\
Potencial pH & 20,96 & 0,07 \\
Sólidos solúveis (SS) & 3427,78 & 12,10 \\
Acidez titulável (AT) & 109,28 & 0,39 \\
SS/AT & 1280,47 & 4,52 \\
\hline
\end{tabular}

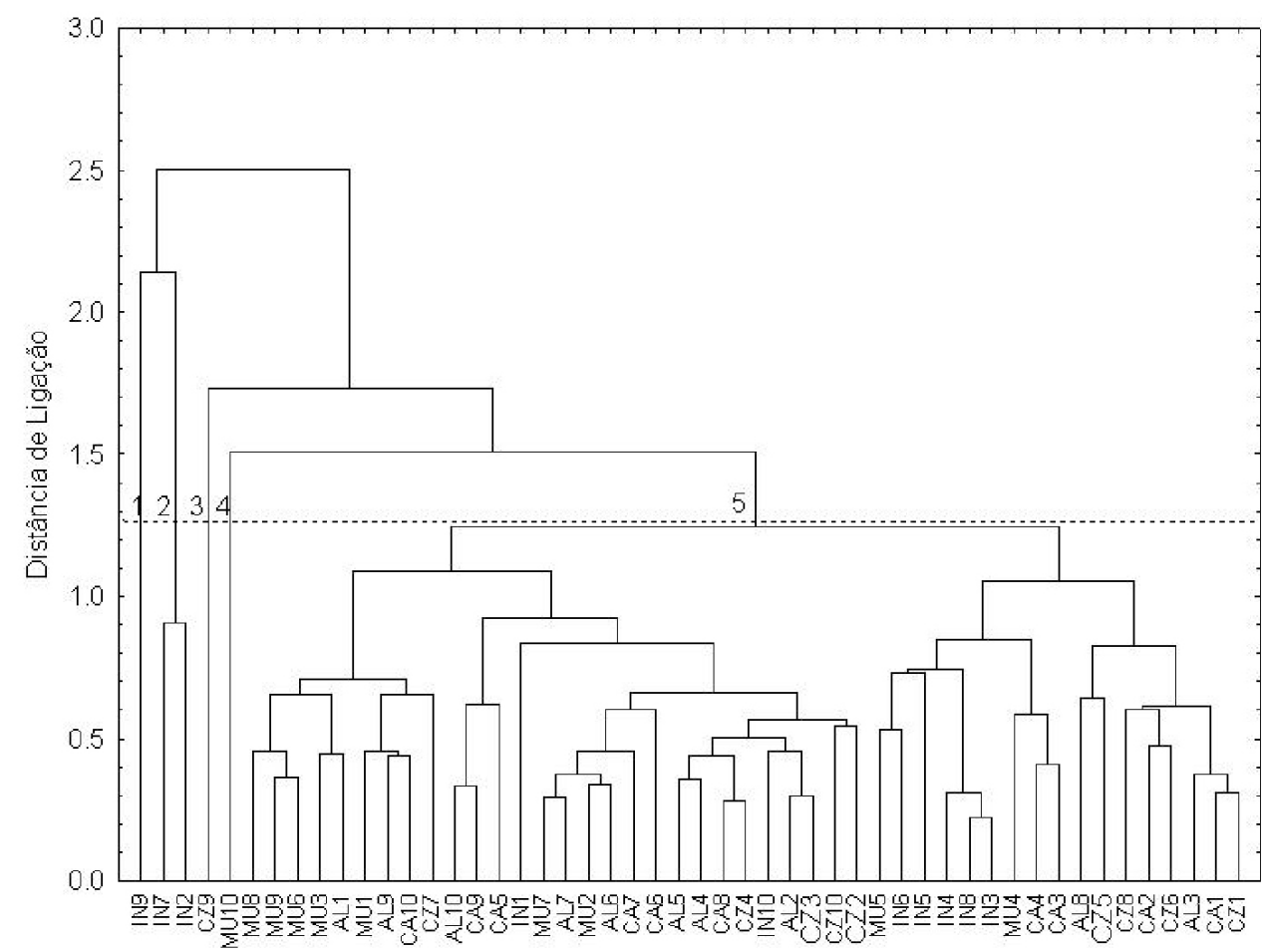

FIGURA 1- Dendrograma de dissimilaridade entre 50 pitangueiras (Eugenia uniflora L.) provenientes de municípios situados no Estado da Bahia. $\mathrm{CCC}=82,70 \%$ 


\section{CONCLUSÕES}

1- Existe variabilidade entre os genótipos de pitangueira, com base na caracterização dos frutos, que pode ser explorada para a conservação e exploração comercial da espécie.

2- Os frutos apresentam características de interesse para exploração comercial com porcentagem de polpa acima de $68 \%$.

3- Os frutos dos genótipos IN9, CZ9, AL3, CA8, CZ5, CA1, AL8, IN1, AL4 e CA2 são os que apresentam as melhores características agronômicas, visando ao aproveitamento industrial.

\section{REFERÊNCIAS}

ARAÚJO. J. M. A. Química de alimentos: teoria e prática. 19. ed. Viçosa: Impressa Universitária, 1995. 335p.

BÁRBARA, M. F. S.; FONSECA, A. A. O.; BASTOS, L. A.; SILVA, L. C. S. Caracterização físicoquímica dos frutos da pitangueira oriundos da região de Santo Antônio de Jesus-BA. In: SIMPÓSIO EM CIÊNCIA E TECNOLOGIA DE ALIMENTOS, 2 ., CONGRESSO DO INSTITUTO NACIONAL DE FRUTOS TROPICAIS, 1., 2010, Aracaju. Anais... Aracaju: Sociedade Brasileira de Tecnologia de Alimentos, 2010. CD-ROM.

BEZERRA, J. E. F.; LEDERMAN, L. E.; SILVA JÚNIOR, J. F. S.; ALVES, M. A. Comportamento da pitangueira (Eugenia uniflora L.) sob irrigação na região do Vale do Rio Moxotó, Pernambuco. Revista Brasileira de Fruticultura, Jaboticabal, v. 26, n. 1, p. 177-179, 2004.

BRASIL. Ministério da Agricultura Pecuária e Abastecimento. Instrução Normativa $n^{\circ} 136$, de 31 de março de 1999. Diário Oficial da República Federativa do Brasil, Brasília, $1^{\circ}$ de abr. Seção 1, p. 25,1999 .

CRUZ, C. D. Aplicação de algumas técnicas multivariadas no melhoramento de plantas. 1990. 188 f. Tese (Doutorado em Genética e Melhoramento de Plantas) - Escola Superior de Agricultura "Luiz de Queiroz", Universidade de São Paulo, Piracicaba, 1990.
CRUZ, C. D. Programa genes (versão Windows): aplicativo computacional em genética e estatística. Viçosa: UFV, 2008.

DAHER, R. F. Diversidade morfológica e isoenzimática em capim-elefante (Pennisetum purpureum Schum). 1993. 110 f. Dissertação (Mestrado em Genética e Melhoramento de Plantas) - Universidade Federal de Viçosa, Viçosa, 1993.

FONSECA, M. D. S.; FONSECA, A. A. O.; PEREIRA, J. M.; SILVA, P. M. Caracterização física dos frutos de genótipos de pitangueira (Eugenia uniflora L.) no município de Cruz das Almas - BA. In: CONGRESSO BRASILEIRO DE MELHORAMENTO DE PLANTAS, 5., 2009, Guarapari-ES. Anais... Guarapari-ES: Sociedade Brasileira de Melhoramento de Plantas, 2009.

INSTITUTO ADOLFO LUTZ. Normas Analíticas do Instituto Adolfo Lutz: métodos químicos e físicos para análises de alimentos. 3.ed. São Paulo: Instituto Adolfo Lutz, 1985, v.1. 371p.

LACERDA, D. R.; ACEDO, M. D. P.; LEMOSFILHO, J. P.; LOVATO, M. B. Genetic diversity and structure of natural populations of Plathymenia reticulata (Mimosoideae), a tropical tree from Brazilian Cerrado. Molecular Ecology, Oxford, v.10, p.1.143-1.152, 2001.

LIMA, V. L. A. G. de; MELO, E. de A.; LIMA, D. E. da S. Fenólicos e carotenoides totais em pitanga. Scientia Agrícola, Piracicaba, v. 59, n.3, p. $447-$ 450, 2002.

LIRA JUNIOR, J. S.; BEZERRA, J. E. F.; LEDERMAN, I. E.; SILVA JÚNIOR, J. Pitangueira. Recife: Empresa de Pesquisa Agropecuária. IPA, 2007. 87 p.

LOPES, A. S. Pitanga e acerola: estudo de processamento, estabilidade e formulação de néctar misto. 2005. 175f. Tese (Doutorado em Tecnologia de Alimentos) - Universidade Estadual de Campinas, Faculdade de Engenharia de Alimentos, Campinas, 2005.

MELETTI, L. M. M.; SOARES-SCOTT, M. D.; PINTO-MAGLIO, C. A.; MARTINS, F. P. Caracterização de germoplasma de maracujazeiro (Passiflora spp.). Revista Brasileira de Fruticultura, Jaboticabal, v. 14, n. 2, p. 157-162, 1992. 
MULAMBA, N. N.; MOCK, J. J. Improvement of yield potential of the Eto Blanco maize (Zea mays L.) population by breeding for plant traits. Egyptian Journal of Genetics and Cytology, Giza, v. 7, p. 40-57, 1978.

OLIVEIRA, F. M. N.; FIGUEIRÊDO, R. M. F.; QUEIROZ, A. J. M. Análise comparativa de polpas de pitanga integral, formulada e em pó. Revista Brasileira de Produtos Agroindustriais, Campina Grande, v. 8, n. 1, p. 25-33. 2006.

OLIVEIRA, L. F. de. Efeito dos parâmetros do processo de desidratação de jaca (Artocarpus heterophyllus Lam.) sobre as propriedades químicas, físico-químicas e a aceitação sensorial. 2009. $121 \mathrm{f}$. Tese (Doutorado em Ciência e Tecnologia de Alimentos) - Instituto de Tecnologia, Departamento de Tecnologia de Alimentos, Universidade Federal Rural do Rio de Janeiro, Seropédica, 2009.

PINTO W. S.; DANTAS, A. C. V. L.; FONSECA, A. A. O.; LEDO, C.A. S.; JESUS, S. C.; CALAFANGE, P. L. P.; ANDRADE, E. M. Caracterização física, físico-química e química de frutos de genótipos de cajazeiras. Pesquisa Agropecuária Brasileira, Brasília, v. 38, n. 9, p. 1.059-1.066, 2003.

SANTOS, L. A. dos. Caracterização de frutos e molecular de umbu-cajazeiras (Spondias sp.) no semiárido da Bahia. 2010. 56f. Dissertação (Mestrado em Recursos Genéticos Vegetais) - Centro de Ciências Agrárias, Ambientais e Biológicas, Universidade Federal do Recôncavo da Bahia, Cruz das Almas, 2010.
SAS INSTITUTE.. SAS/STAT ${ }^{\circledR}$ user's guide. $4^{\text {th }}$ ed. North Carolina, 1989. v.2, 846 p.

SINGH, D. The relative importance of characters affecting genetic divergence. The Indian Journal of Genetic and Plant Breeding, New Delhi, v. 41, p. 237-245, 1981.

SNEATH, P. H.; SOKAL, R. R. Numerical taxonomy: the principles and practice of numerical classification. San Francisco: W.H. Freeman, 1973. $573 \mathrm{p}$.

STATSOFT. Statistica for Windows (data analysis software system), version 7.1. Tulsa, 2005.

VAZ PATTO, M.C.; SATOVIC, Z.; PÊGO, S.; FEVEREIRO, P. Assessing the genetic diversity of Portuguese maize germplasm using microsatellite markers. Euphytica, Wageningen, v.137, p.63-72, 2004.

WEILER, R. L. Caracterização morfológica, citogenética e molecular de uma população de tangerineiras híbridas de 'Clementina Fina' (Citrus clementina Hort. ex Tan.) e 'Montenegrina' (Citrus deliciosa Ten.). 2006. 78 f. Dissertação (Mestrado em Fitotecnia) - Faculdade de Agronomia, Universidade Federal do Rio Grande do Sul, Porto Alegre, 2006. 